Liver, Pancreas and Biliary Tract

\title{
Different biochemical patterns in type II and type III mixed cryoglobulinemia in HCV positive patients
}

\author{
Umberto Basile ${ }^{\mathrm{a}, 1}$, Francesca Gulli ${ }^{\mathrm{b}, 1}$, Laura Gragnani ${ }^{\mathrm{c}, *}$, Krizia Pocino ${ }^{\mathrm{a}}$, \\ Cecilia Napodano ${ }^{a}$, Luca Miele $^{\mathrm{d}}$, Stefano Angelo Santini ${ }^{\mathrm{a}}$, Mariapaola Marino ${ }^{\mathrm{e}}$, \\ Anna Linda Zignego ${ }^{c}$, Gian Ludovico Rapaccini ${ }^{\mathrm{d}}$ \\ a Department of Diagnostic Imaging and Laboratory Medicine, Fondazione Policlinico Universitario Agostino Gemelli - Università Cattolica del Sacro Cuore, \\ Rome, Italy \\ ${ }^{\mathrm{b}}$ Department of Laboratory Medicine - Madre Giuseppina Vannini Hospital, Rome, Italy \\ ${ }^{\mathrm{c}}$ Center for Systemic Manifestations of Hepatitis Viruses (MaSVE), Department of Experimental and Clinical Medicine, University of Florence, Florence, Italy \\ ${ }^{\mathrm{d}}$ Institute of Internal Medicine, Fondazione Policlinico Universitario Agostino Gemelli - Università Cattolica del Sacro Cuore, Rome, Italy \\ e Institute of General Pathology, Fondazione Policlinico Universitario Agostino Gemelli - Università Cattolica del Sacro Cuore, Rome, Italy
}

\section{A R T I C L E I N F O}

\section{Article history:}

Received 29 November 2017

Received in revised form 16 March 2018

Accepted 19 March 2018

Available online 4 April 2018

\section{Keywords:}

Anti nuclear antibody (ANA)

Cryoglobulins

$\mathrm{HCV}$

$\operatorname{IgG3}$

Rheumatoid factor

\begin{abstract}
A B S T R A C T
Background: Reversible cryoprecipitability of proteins is observed as a concomitant feature of immune complex formation. Mixed cryoglobulinemia (MC) is systemic vasculitis, associated with mixed IgM and IgG cryoglobulins (CGs) showing rheumatoid factor (RF) activity. It is frequently associated with hepatitis $\mathrm{C}$ virus (HCV). This study investigates the presence of IgG RF and anti-nuclear antibodies (ANA) in cryoprecipitates of patients with type III and type II MC, to understand the biochemical patterns associated with different types of MC to a greater degree.

Methods: Sera from $70 \mathrm{HCV}$ untreated patients with type III or type II MC were tested by immunofixation for IgG3 and through ELISA for IgG RF. Cryoprecipitates were analysed for ANA by indirect immunofluorescence to identify specific patterns.

Results: After stratification according to MC type, the ANA patterns between type II and type III MC were statistically different. IgG3 levels and IgG-RF positivity were significantly higher in type III cryoprecipitate. We observed a higher positivity of IgG3 and a significant difference between the liver fibrosis stage, ANA and IgG-RF in the cryoprecipitate.

Conclusion: Results show a combination of biochemical markers and autoantibodies associated to mixed cryoglobulinemia; these findings could be further investigated in order to ascertain their usefulness in assessing the risk for the development of mixed cryoglobulinemia.
\end{abstract}

(c) 2018 Editrice Gastroenterologica Italiana S.r.l. Published by Elsevier Ltd. All rights reserved.

\section{Introduction}

Cryoglobulinemia is defined as the presence of immunoglobulins (Igs) precipitating in serum at a temperature of $<37^{\circ} \mathrm{C}$ and dissolving upon reheating of the specimen. Reversible cryoprecipitability of proteins may be observed as a concomitant feature of immune complex formation. The relevance of the cryoprecipitation phenomenon became apparent when clinical associations with vas-

\footnotetext{
* Corresponding author at: Center for Systemic Manifestations of Hepatitis Viruses (MASVE), Department of Experimental and Clinical Medicine, University of Florence, Largo Brambilla 3, 50134 Florence, Italy.

E-mail address: laura.gragnani@unifi.it (L.Gragnani).

1 Coauthor.
}

culitis and nephritis, resembling those seen in experimental serum sickness, were described [1].

Cryoglobulins (CGs) were classified by Brouet et al. [2] into three types according to the characteristics of the constituting Ig: type I refers to the presence of a single monoclonal Ig; type II is formed by two Igs, one monoclonal and the other polyclonal. Frequently, a monoclonal IgM-rheumatoid factor (RF) complexed with polyclonal IgG; type III is composed of polyclonal IgM-RF and the corresponding antigen (usually polyclonal IgG) immunocomplex. The last two types are referred to as mixed cryoglobulins (MCs). CGs in vivo precipitate in the thin vessels of the extremities and the skin, where the blood temperature can drop well below $37^{\circ} \mathrm{C}$, as well as in the kidney. Type II and type III CGs were defined as "essential" as they were found in patients without any clinically apparent disease [3]. 
Table 1

Main characteristics of the study population.

\begin{tabular}{|c|c|c|c|c|}
\hline & \multirow[b]{2}{*}{ Total } & \multicolumn{2}{|c|}{ Type of mixed cryoglobulinemia } & \multirow[t]{2}{*}{$\mathrm{p}$} \\
\hline & & Type II & Type III & \\
\hline Total & 70 & $30(43 \%)$ & $40(578 \%)$ & \\
\hline Age (years) ${ }^{a}$ & $64 \pm 11.97$ & $69.4 \pm 10$ & $60 \pm 411.8$ & \\
\hline Gender (male/female) & $24 / 46$ & October-20 & $14 / 26$ & \\
\hline \multicolumn{5}{|l|}{ Metavir score ${ }^{b}$} \\
\hline F0 & 13 & 0 & 13 & \\
\hline $\mathrm{F} 1$ & 20 & 17 & 3 & \\
\hline $\mathrm{F} 2$ & 28 & 7 & 21 & \\
\hline F3 & 4 & 1 & 3 & \\
\hline F4 & 5 & 5 & 0 & \\
\hline \multicolumn{5}{|l|}{ HCV genotype } \\
\hline 1 & $1(1.4 \%)$ & $1(3.3 \%)$ & - & \\
\hline $1 \mathrm{a}$ & $13(18.6 \%)$ & $1(3.3 \%)$ & $12(30 \%)$ & \\
\hline $1 b$ & $34(48.6 \%)$ & $16(53.3 \%)$ & $18(45 \%)$ & \\
\hline $2 \mathrm{a}$ & $8(11.4 \%)$ & - & $8(20 \%)$ & \\
\hline $2 a / 2 c$ & $5(17.1 \%)$ & $5(16.7 \%)$ & - & \\
\hline $2 a-3 c$ & $2(2.8 \%)$ & - & $2(5 \%)$ & \\
\hline 3 & $1(1.4 \%)$ & $1(3.3 \%)$ & - & \\
\hline 3a & $2(2.8 \%)$ & $2(6.6 \%)$ & - & \\
\hline \multicolumn{5}{|l|}{ MC symptoms } \\
\hline None & $19(27.1 \%)$ & - & $19(47.5 \%)$ & \\
\hline Neuropathy & $25(35.7 \%)$ & $18(60 \%)$ & $7(17.5 \%)$ & \\
\hline Meltzer's triad & $30(42.8 \%)$ & $20(66.7 \%)$ & $10(25 \%)$ & \\
\hline Renal involvement & $5(7.1 \%)$ & $3(10 \%)$ & $2(5 \%)$ & \\
\hline Raynaud phenomenon & $12(17.1 \%)$ & $11(36.5 \%)$ & $1(2.5 \%)$ & \\
\hline \multicolumn{5}{|l|}{$\operatorname{ALT}(U / L)^{c}$} \\
\hline $7-45 \mathrm{U} / \mathrm{L}$ & $13(19 \%)$ & $13(43.3 \%)$ & - & $\mathrm{p}<0.001$ \\
\hline$>45 \mathrm{U} / \mathrm{L}$ & $57(81 \%)$ & $17(56.6)$ & 40 (100\%) & Type II vs. type III \\
\hline
\end{tabular}

a Data is expressed as mean \pm standard deviation.

b Based on liver stiffness assessed by FibroScan.

c ALT normal range: 7-45 U/L.

MC syndrome is a systemic vasculitis, affecting the small arteries and veins, associated with the presence of large amounts of mixed IgM and IgG cryoglobulins, showing RF activity. It is frequently associated with hepatitis $C$ virus (HCV) infection [4-6].

HCV chronic infection is characterized by the possible development of both hepatic and extrahepatic manifestations, and it is responsible for poly-oligoclonal B-lymphocyte expansion, leading to several immune-mediated disorders. HCV-infected patients predisposition to develop MC vasculitis remains unclear, but the host's immune response genes may play a role [5].

In 1992, Musset et al. observed microheterogeneity characterized by the presence of two or more monoclonal IgM or IgG [7-9] in patients with CGs.

Type III pattern CG is suggested to be a transitional state that evolves from a polyclonal population of B-cells to an oligoclonal, and finally to a monoclonal type II CG [9].

Persistent HCV infection associated with MC shows serological features of an autoimmunity process. Presence of non-organspecific autoantibodies including antinuclear antibodies (ANA) is well established, and is a peculiar characteristic of Systemic Autoimmune-Rheumatic Diseases, although their pathogenic role has not been uncovered yet [10]. As serologic biomarkers, ANAs are useful for diagnosing patients with autoimmune or connective tissue diseases, but are also associated with HCV-induced responses $[11,12]$.

HCV infection may be considered as a multiple stage disease and it shows a correlation between IgG subclasses and the presence of IgG-RF, and ANA in HCV with type III CGs [13]. IgG3 presence in cryoprecipitates type III in HCV- and ANA-positive patients would constitute a decisive factor for the possible activation of autoimmune mechanisms in the long term [13].

IgG3 are known to be autoreactive clones and their ability to activate several cell clones is confirmed by many clinical studies [14].
Considering the increased risk of immunological disorders in patients with $\mathrm{HCV}$, it is necessary to determine sensitive and specific sets of biomarkers. This study aims to investigate the presence of IgG RF and ANAs in cryoprecipitates of type III and type II CGs, as well as to search for a relationship between their occurrence in $\mathrm{HCV}$-positive patients and HCV-related mixed cryoglobulinemia.

\section{Materials and methods}

\subsection{Patients}

CGs samples were collected from 70 patients with HCV infection. Patients were enrolled at two Italian centers (Center for Systemic Manifestations of Hepatitis Viruses (MaSVE), Department of Experimental and Clinical Medicine, University of Florence, Florence, Italy and Fondazione Policlinico Agostino Gemelli Università Cattolica Del Sacro Cuore, Rome, Italy).

Subjects were included in the study according to the following criteria: presence of HCV-RNA in the serum, absence of antiviral treatment and/or immunosuppressive therapy, and the presence of mixed cryoglobulinemia symptoms (Table 1 ).

MC was diagnosed when the presence of serum CGs was found in at least two metachronous tests. ALT values were available for all the patients and the METAVIR score, was assessed by transient elastography (Fibroscan). Exclusion criteria was: the presence of co-infections (HIV and HBV), ongoing drug treatment and the presence of other autoimmune disorders. Written informed consent was obtained from all individuals and the study was conducted in accordance with the Declaration of Helsinki.

\subsection{Clinical and laboratory assessment}

Twenty ml of peripheral blood was collected using tubes without anticoagulant, and kept at $37^{\circ} \mathrm{C}$ both before and after sample collection for at least 30 min until complete clotting $[15,16]$. 
$\mathbf{A}$

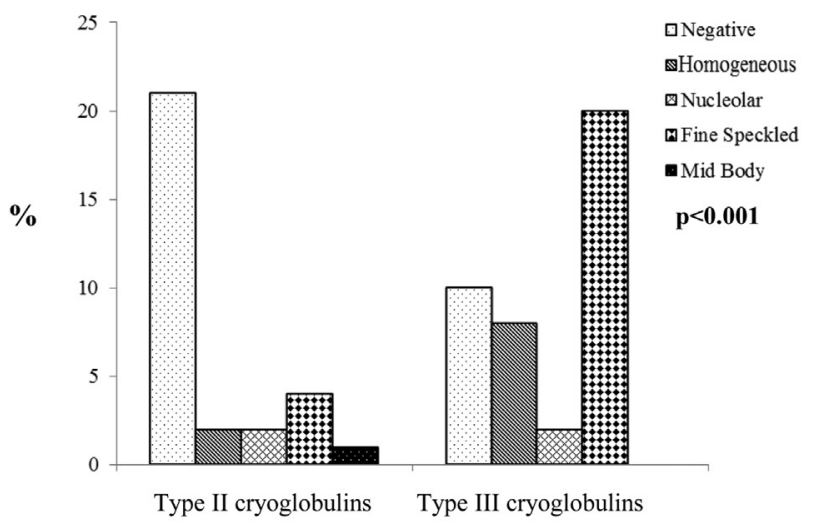

C

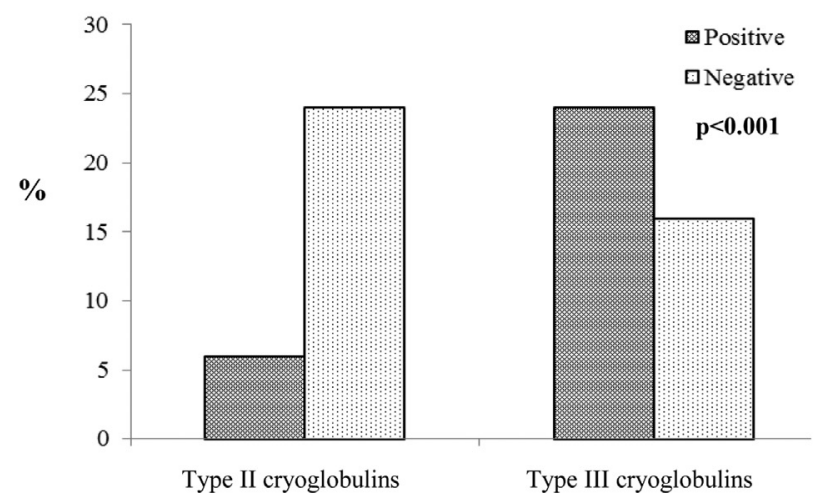

B

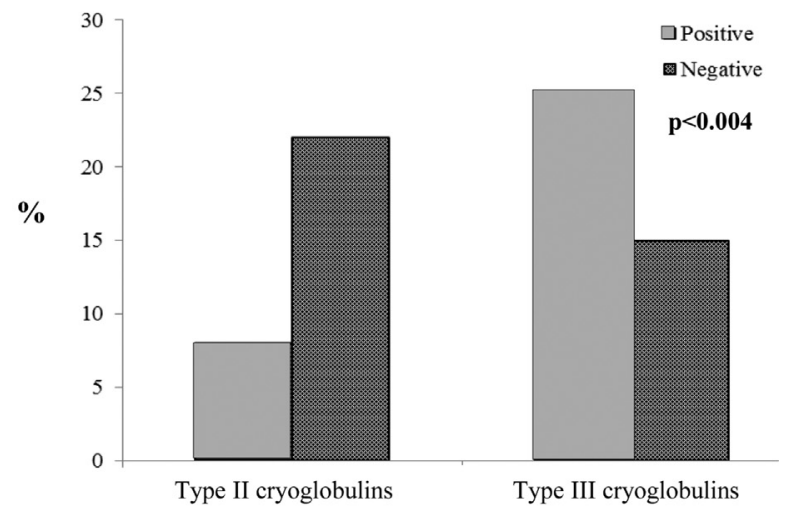

D

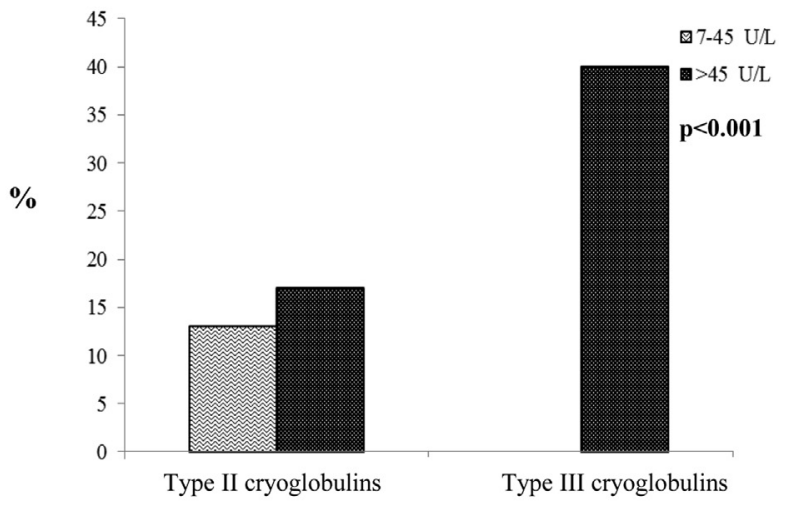

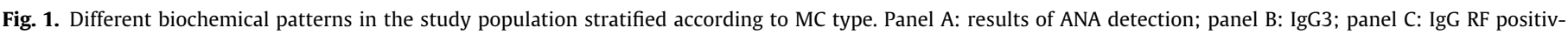
ity/negativity; panel D: ALT normal level (7-45U/L) and abnormal level (>45 U/L) distribution.

This implies a simple biochemical quantification, which nevertheless requires strict pre-analytical protocol adhesion in order to maintain the sample at a stable temperature of $37^{\circ} \mathrm{C}$, especially throughout the initial steps of the test [17].

Following centrifugation, the supernatant serum samples were transferred into Wintrobe tubes and incubated at $4{ }^{\circ} \mathrm{C}$. The precipitation process manifests itself in a variety of ways among samples, depending on the concentration of CGs present, and may require either a few hours (when CGs-often type I-become insoluble at room temperature) or longer periods of time (7 days), particularly in the event of low concentrations of type III CGs. Cryocrit percentage was assessed, the supernatant was removed, and the remaining cryoprecipitate was recovered and washed 3 times [16]. Washing was carried out by using a $4 \%$ polyethylene glycol 6000 (PEG 6000) solution obtained by mixing $4 \mathrm{~g}$ of PEG 6000 in $100 \mathrm{ml}$ of phosphate buffer solution (PBS) (4\%p/v). $2 \mathrm{ml}$ of $4 \%$ PEG solution was added to each cryoprecipitate sample, which was then centrifuged at $3000 \times g$ for $5 \mathrm{~min}$ at $37^{\circ} \mathrm{C}$. Cryoprecipitate was redissolved in the appropriate volume of a 3\% PEG 6000 solution, and re-solubilized for $30 \mathrm{~min}$ at $37^{\circ} \mathrm{C}$. Igs were typed and characterized by immunofixation electrophoresis (IFE) on the G26 Fully automated system (Interlab, Italy), according to the manufacturer's instructions. IFE was performed using antisera against $\gamma, \alpha, \mu, \kappa$, and $\lambda$. IgG 3 subclasses were characterized by means of antisera (Binding Site, UK). A stained band in the IFE represents a reaction between an antiserum and a specific protein on the specimen. IgG $\mathrm{RF}$ was tested using ELISA kits for quantitative measurement of IgG $\mathrm{RF}$ (INOVA, USA). An aliquot of the sample was previously removed for ELISA testing, and assayed at $37^{\circ} \mathrm{C}$. All samples were analysed at the same time following the manufacturer's instructions, and plates were immediately read on a plate reader as indicated by the manufacturer. Following Clinical and Laboratory Standards Institute (CLSI) guidelines [18], we tested 20 healthy donors from the local population to verify adherence to CLSI EP 28A3C [18] and according to the manufacturer, IgG $R F \geq 9$ was to be used as cut-off positive.

ANA determination was carried out by means of indirect immunofluorescence (IIF) on HEp-2 cells, American Type Culture Collection (ATCC) (INOVA, USA), which enables the expression of antigens throughout all stages of the cell cycle [19]. Cells were cultured on glass slides in multiple well plates and the appropriate titer of cryoprecipitate was added to cells. Slides were incubated for $30 \mathrm{~min}$ and then washed with PBS in order to remove any non-specific binding or excess. A secondary polyclonal antibody (anti-human IgG polyclonal sheep antiserum, FITCconjugated anti-IgG anti-serum, Invitrogen, Life Technologies) was subsequently added. Excess conjugated secondary antiserum was removed by washing with PBS solution. Slides were fixed with glycerol and mounted for microscopy. Cryoprecipitate dilution for ANA detection was in PBS 1:5. This is currently the one indication concerning adequate titers to be used for ANA detection in cryoprecipitates [13].

Quantitative HCV-RNA detection in cryoprecipitate was determined by a routine method and a virus genotype was determined for each sample (Siemens Healthcare, Germany). Presence and quantification of HCV-RNA were determined by means of real- 
A

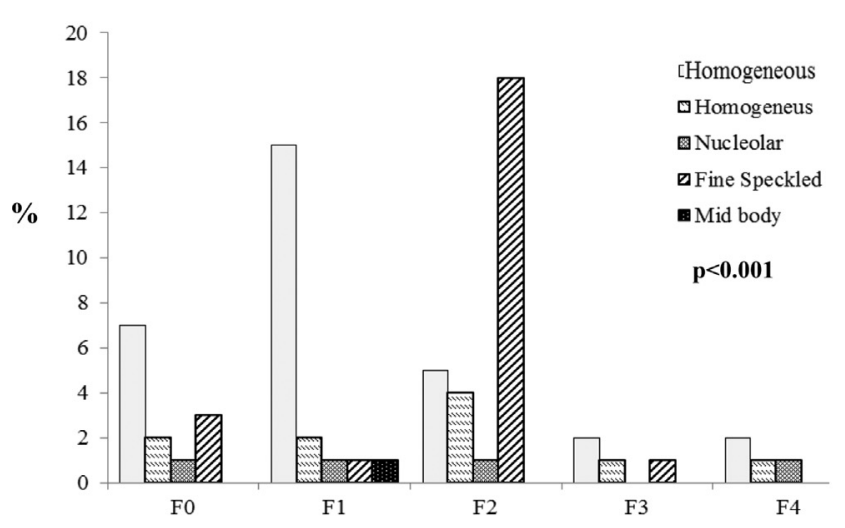

C

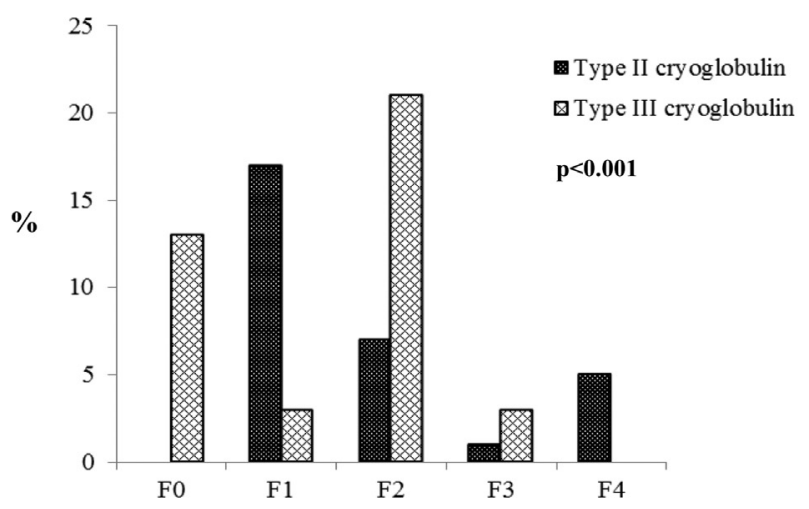

B

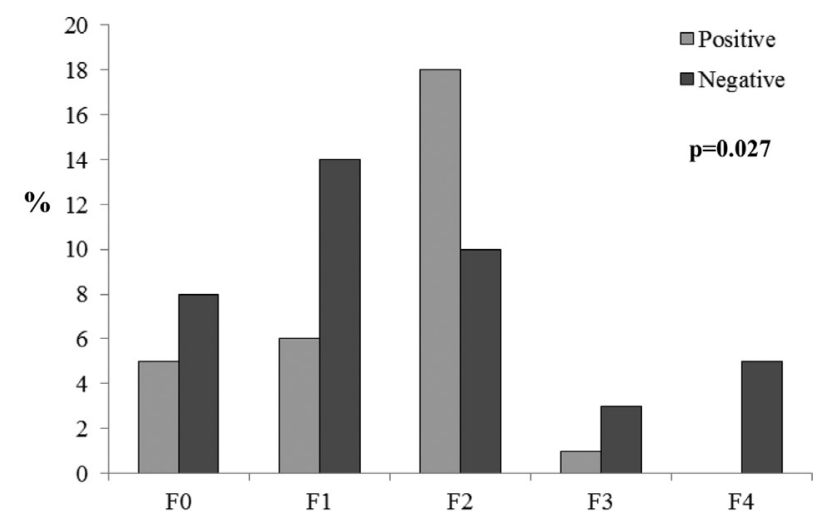

D

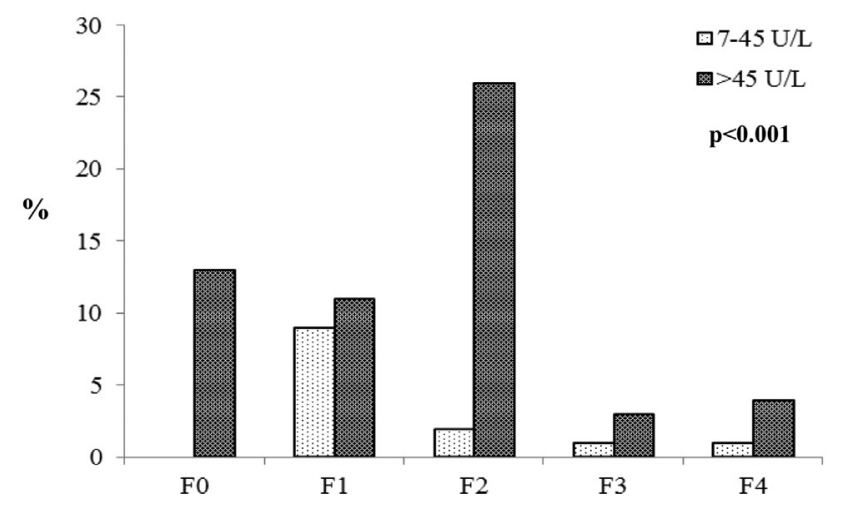

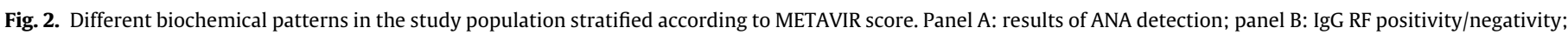
panel C: MC type distribution; panel D: ALT normal level (7-45 U/L) and abnormal level (>45 U/L) distribution.

time polymerase chain reaction (PCR), transcription-mediated amplification (TMA), and multi-probe reverse hybridization of the 5'-untranslated region (5'-UTR) of the HCV genome. Testing for HCV genotype and subtype was performed through reverse transcription and PCR and reverse hybridization of the HCV genome.

\subsection{Statistical analysis}

Statistical analysis was performed using the Statistical Package for Social Science (SPSS) version 15.0 (Chicago, Illinois). Distribution of the data in the groups was preliminarily evaluated via the Kolmogorov-Smirnov test. Continuous variables were presented as mean $\pm S D$. Categorical variables were described as numbers in percentage and compared using the $\chi^{2}$ test. The differences in molecule levels between groups were analyzed using the Kruskal-Wallis, Mann-Whitney Test, and multiple comparisons post hoc tests as appropriate. A two-sided $p$ value of less than 0.05 was considered, associated with statistical significance.

\section{Results}

A total of 70 patients, 46 female and 24 male, mean age 64 years (range 35-79) were retrospectively enrolled. IgG-RF was present in $42.9 \%(\mathrm{~N}=30)$ of patient cryoprecipitates. The IgG3 subclass was negative in $37 / 70(53 \%)$ patients.

ANA determination assay was negative in $32 / 70$ subjects (46\%) and positive in the remaining 38/70 (54\%); ALT levels were normal (mean $29.9 \pm 9.8 \mathrm{U} / \mathrm{L}$ ) in $13 / 70$ patients $(19 \%)$ and increased (mean $106.1 \pm 52.7 \mathrm{U} / \mathrm{L}$ ) in $57 / 70(81 \%$ ), normal values range from 7 to $45 \mathrm{U} / \mathrm{L}$.

Results of cryoglobulin type assessment showed that 30/70 patients (43\%) had type II and 40 had type III MC (57\%). Stratifying results according to MC type, the ANA patterns were statistically different between type II and type III MC $(\mathrm{p}<0.001)$ (Fig. 1, panel A); IgG3 levels and IgG-RF positivity were higher in type III cryoprecipitate $(p<0.004$, Fig. 1, panel B and $p<0.001$ panel C respectively); ALT levels were also significantly different between type II and type III MC ( $\mathrm{p}<0.001)$ : in the type II MC group $13 / 30$ patients $(43 \%)$ had normal ALT (mean 29.9 $\pm 9.8 \mathrm{U} / \mathrm{L}$ ) and $17 / 30$ (57\%) had an abnormal ALT level (mean $84.3 \pm 43.4 \mathrm{U} / \mathrm{L}$ ); all type III MC patients had an abnormal ALT value (mean $115.4 \pm 53.9 \mathrm{U} / \mathrm{L}$ ) (Fig. 1, panel D). Dividing the total population into two groups, according to ALT levels (normal and abnormal), we did not find a significant correlation with IgG3 positivity ( $\mathrm{p}=0.056$; data not shown).

Stratifying for the liver fibrosis stage, according to the METAVIR scoring system, we observed a higher positivity of IgG3 and, a statistically significant difference between the stage of liver fibrosis and ANA ( $p<0.001$, Fig. 2, panel A), IgG-RF in the cryoprecipitate ( $p=0.027$; Fig. 2, panel $B$ ) and also a difference in MC type frequency $(\mathrm{p}<0.001)$ (Fig. 2, panel C). ALT levels stratified according to liver fibrosis stages are shown in Fig. 2, panel D.

\section{Discussion}

The pathogenesis of HCV-related MC is still not completely known [20]. Key factors are probably chronic stimulation of the 
immune system through $\mathrm{HCV}$ infection, and the dysfunction of the reticuloendothelial system [21], thereby leading to the production and persistence of antibodies that are able to precipitate below body temperature [22]. There is a higher prevalence of IgG3 responses to HCV antigens in HCV-related MC patients than in $\mathrm{HCV}$ patients without MC [23].

IgG3 fixes complement more efficiently than other subclasses, thereby leading to the activation of the classical pathway $[13,24]$. CGs are heterogeneous immune-complexes, and do not always correlate with the severity of symptoms, [25].

HCV patients express a great variety of autoantibodies usually at a low titer [26], and they may be associated with underlying autoimmune disorders or liver inflammation in HCV infection. A possible reason for antibody production is over activation and proliferation of B lymphocytes, via interaction with the HCV surface protein [27].

Data shows a different presence of autoantibodies and IgG3 in type II and type III CRGs that probably represents a modulation of the immune system to continuous antigenic stimulation.

In our cohort, we analysed if there was any correlation between type II and type III MC with the presence of ANA, IgG-RF, IgG3 subclass and ALT elevation (Fig. 1).

The difference between the two groups is probably due to the existence of a progressive evolution of CGs. Elevated ALT levels in type III are probably due to a broader immune response that involves both $\operatorname{IgG} 1$, which take part in the recognition of the virus, and the presence of IgG3 that triggers some autoimmune responses as well as IgG-RF that participates in the formation of the immune complex [15]. It is also conceivable that the presence of ANA and then autoreactive clones represents the trigger for extrahepatic autoimmune manifestations.

Type II CGs arise by a later clonal selection characterized by the presence of a monoclonal $\kappa-\operatorname{IgM}$ responsible for typical MC symptoms. Low temperatures and IgG3 seem to trigger a reversible cryoprecipitation, possibly by inducing steric modifications of Ig molecules [28]. The biological activities of each subclass of IgG are not fully known. IgG receptors are strikingly numerous in humans. They are comprised of high-affinity and low-affinity. The presence of IgG3 in cryoprecipitates of HCV- and ANA-positive patients constitutes a decisive factor for the possible activation of autoimmune mechanisms in the long term [15]. In addition, IgG3 positive patients are also positive for IgG-RF, known to be autoreactive clones, and their capacity to activate several cell clones. Thus, the presence of IgG3 in cryoprecipitates may suggest a more highly activated immune system, which is then more exposed to the mechanisms of autoimmune diseases [15]. The possible presence of IgG-RF or ANA may be the result of selected clones of autoreactive IgG in HCV patients.

Hepatic fibrosis stimulates the production and accumulation of collagen and extracellular matrix proteins. Molecules derived from both pathogen and host can induce inflammation in a rigidly controlled process, that engages immune cells and parenchymal cells via different signal transduction pathways [29].

In the present study patients with Metavir score F2, had a type III CG pattern. All patients with a type III CG pattern had elevated ALT. The elevated ALT also correlated with the presence of IgG-RF in the cryoprecipitate and particularly fine speckled ANA (Fig. 2 panel D). These findings were unexpected, and further studies will be useful in confirming if a mild fibrosis stage with high ALT levels is correlated with a "secretory" behavior, high levels of cryoprecipitate, and ANA positivity.

Results show that a combination of biochemical markers could have predictive value for the diagnosis of clinically significant extrahepatic manifestations in patients with CG, and detection of autoantibodies has a significant role in the mosaic of autoimmunity as an important risk factor for the development of autoimmune disease [30]. Considering the double-edged nature of antibodies, unveiling the origin and pathological implications of infectiondriven autoantibodies could be a milestone in the development of effective therapeutic strategies against both chronic infections and autoantibody-mediated systemic disorders. Moreover, the hypothesis that IgG3 could play an important role both in the induction and persistence of cryoglobulinemia, as well as the assumption of an evolution of such a condition from type III CRGs to type II CRGs, have been confirmed by other authors as well [28].

The presence of autoantibodies in the course of chronic HCV infection (in particular ANA) is a phenomenon that could be a virus-induced autoimmunity, of which cryoglobulinemia could be a possible model. The detection of an autoantibody is not necessarily related to a frank autoimmune disease, but the presence of ANA could be a key factor in autoimmune mechanisms activation and, their detection could be a screening test, and a basic tool in the diagnosis and management of patients with autoimmune disease.

In conclusion, HCV disease is the result of a multifactorial and multistep biochemical and pathogenetic process. Our results, along with longitudinal studies, demonstrate that the discussed set of biomarkers can be used as a predictive tool for variations in clinical manifestations. Some extrahepatic manifestations are immune mediated while others seem to be driven by chronic inflammation. Also, the availability of these non-invasive tests could be valuable in order to assess the effective control of HCV-related lymphoproliferations or autoimmune diseases, after viral eradication, highlighting an area of interest that may warrant further investigation, in order to determine variations in cryoprecipitates associated with different outcomes in HCV patients.

\section{Conflict of interest}

None declared.

\section{Guarantor}

UB.

\section{References}

[1] Ferri C, Zignego AL, Pileri SA. Cryoglobulins. J Clin Pathol 2002;55(1):4-13.

[2] Brouet JC, Clauvel JP, Danon F, Klein M, Seligmann M. Biologic and clinical significance of cryoglobulins. A report of 86 cases. AM J Med 1974;57:775-88.

[3] Meltzer M, Franklin EC. Cryoglobulinemia: a study of 29 patients. Am J Med 1966;40:828-36.

[4] Ferri C, Sebastiani M, Giuggioli D, Colaci M, Fallahi P, Piluso A, et al. Hepatitis $C$ virus syndrome: a constellation of organ- and non-organ specific autoimmune disorders, B-cell non-Hodgkin's lymphoma, and cancer. World J Hepatol 2015;7(March (3)):327-43.

[5] Cacoub P, Gragnani L, Comarmond C, Zignego AL. Extrahepatic manifestations of chronic hepatitis C virus infection. Dig Liver Dis 2014;46S5:S165-73.

[6] Zignego AL, Gragnani L, Piluso A, Sebastiani M, Giuggioli D, Fallahi P, et al. Virusdriven autoimmunity and lymphoproliferation: the example of HCV infection. Expert Rev Clin Immunol 2015;11(1):15-31.

[7] Musset L, Diemert MC, Taibi F, Thi Huong Du L, Cacoub P, Leger JM, et al. Characterization of cryoglobulins by immunoblotting. Clin Chem 1992;38:798-802.

[8] Tissot JD, Pietrogrande M, Testoni L, Invernizzi F. Clinical implications of the types of cryoglobulins determined by two dimensional polyacrylamide gel electrophoresis. Haematologica 1998;83:693-700.

[9] Ponten F, Halimi C, Brocard A, Delacour T. Biclonal immunoglobulin M dysglobulinaemia: evolving aspects in a case of primary Sjögren's syndrome. Eur J Clin Chem Clin Biochem 1997;35:287-90.

[10] Agmon-Levin N, Damoiseaux J, Kallenberg C, Sack U, Witte T, Herold M, et al. International recommendations for the assessment of autoantibodies to cellular antigens referred to as anti-nuclear antibodies. Ann Rheum Dis 2014;73:17-23.

[11] Zusinaite E, Metskula K, Salupere R. Autoantibodies and hepatitis C virus genotypes in chronic hepatitis C patients in Estonia. World J Gastroenterol 2005;11:488-91.

[12] Chrétien P, Chousterman M, Abd Alsamad I, Ozenne V, Rosa I, Barrault C, et al. Non-organ-specific autoantibodies in chronic hepatitis $C$ patients: association with histological activity and fibrosis. J Autoimmun 2009;32:201-5.

[13] Basile U, Gulli F, Torti E, De Matthaeis N, Colacicco L, Cattani P, et al. Antinuclear antibody detection in cryoprecipitates: distinctive patterns in hepatitis C virus-infected patients. Dig Liver Dis 2015;47:50-6. 
[14] Izui S, Berney T, Shibata T, Fulpius T. IgG3 cryoglobulins in autoimmune MRLlpr/lpr mice: immunopathogenesis, therapeutic approaches and relevance to similar human diseases. Ann Rheum Dis 1993;52(Suppl 1):S48-54.

[15] Bakker AJ, Slomp J, de Vries T, Boymans DA, Veldhuis B, Halma K, et al. Adequate sampling in cryoglobulinaemia: recommended warmly. Clin Chem Lab Med 2003;41:85-9.

[16] Kallemuchikkal U, Gorevic PD. Evaluation of cryoglobulins. Arch Pathol Lab Med 1999;123:119-25

[17] Basile U, Torti E, Dell'Abate MT, Colacicco L, Gulli F, Zuppi C, et al. Pre-analytical phase in cryoglobulin (CRG) detection: an alternative method for sample transport. Clin Chem Lab Med 2016:54(4):e123-6.

[18] Clinical and Laboratory Standards Institute. EP05-A3 evaluation of precision of quantitative measurement procedures: approved guideline. 3rd ed. Wayne, PA 19087 USA: Clinical Laboratory Standards Institute; 2014.

[19] Meroni PL, Schur PH. ANA screening: an old test with new recommendations. Ann Rheum Dis 2010;69:1420-2.

[20] Zignego AL, Ramos-Casals M, Ferri C, Saadoun D, Arcaini L, Roccatello D, et al. International therapeutic guidelines for patients with HCV-related extrahepatic disorders. A multidisciplinary expert statement. Autoimmun Rev 2017;16:523-41.

[21] Minopetrou M, Hadziyannis E, Deutsch M, Tampaki M, Georgiadou A, Dimopoulou E, et al. Hepatitis C virus (HCV)-related cryoglobulinemia: cryoglobulin type and anti-HCV profile. Clin Vaccine Immunol 2013;20(May (5)):698-703.

[22] Gulli F, Basile U, Gragnani L, Fognani E, Napodano C, Colacicco L, et al. Autoimmunity and lymphoproliferation markers in naïve HCV-RNA positive patients without clinical evidence of autoimmune/lymphoproliferative disorders. Dig Liver Dis 2016;48(August (8)):927-33.
[23] Otani M, Kuroki A, Kikuchi S, Kihara M, Nakata J, Ito K, et al. Sialylation determines the nephritogenicity of IgG3 cryoglobulins. J Am Soc Nephrol 2012;23(November (11)):1869-78.

[24] Dispenzieri A, Gorevic PD. Cryoglobulinemia. Hematol Oncol Clin North Am 1999;13:1315-49.

[25] Christodoulou DK, Dalekos GN, Merkouropoulos MH, Kistis KG, Georgitsi G, Zervou E, et al. Cryoglobulinemia is not a major problem in clinical practice due to chronic viral hepatitis infections. Eur J Intern Med 2001;12:435-41.

[26] Lunel F. Hepatitis C virus and autoimmunity: fortuitous association or reality? Gastroenterology 1994;107:1550-5.

[27] Yang DH, Ho LJ, Lai JH. Useful biomarkers for assessment of hepatitis $\mathrm{C}$ virus infection-associated autoimmune disorders. World J Gastroenterol 2014;20(11):2962-70.

[28] Basile U, Gulli F, Gragnani L, Fognani E, Napodano C, Pocino K, et al. IgG3 subclass: A possible trigger of mixed cryoglobulin cascade in hepatitis $\mathrm{C}$ virus chronic infection. Dig Liver Dis 2017;49:1233-9.

[29] Szabo G, Petrasek J. Inflammasome activation and function in liver disease. Nat Rev Gastroenterol Hepatol 2015;12(July (7)):387-400.

[30] Shoenfeld Y, Blank M, Abu-Shakra M, Amital H, Barzilai O, Berkun Y, et al. The mosaic of autoimmunity: prediction, autoantibodies, and therapy in autoimmune diseases - 2008. Isr Med Assoc J 2008;10:13-9. 